\title{
Substantia Gelatinosa
}

National Cancer Institute

\section{Source}

National Cancer Institute. Substantia Gelatinosa. NCI Thesaurus. Code C12641.

A collection of gelatinous-appearing grey matter cells in the dorsal horn of the spinal cord that receives direct input from the dorsal nerve roots and plays a role in regulating pain impulses at the spinal cord level. 